\title{
Genetic rationale for microheterogeneity of human diphosphoinositol polyphosphate phosphohydrolase type 2
}

\author{
James J. Caffrey ${ }^{\mathrm{a}}$, Stephen B. Shears ${ }^{\mathrm{b}, *}$ \\ ${ }^{a}$ InforMax Inc., 7600 Wisconsin Ave., Bethesda, MD 20814, USA \\ ${ }^{\mathrm{b}}$ Inositide Signaling Group, National Institute of Environmental Health Sciences, NIH, Research Triangle Park, PO Box 12233, NC 27709, USA
}

Received 29 November 2000; accepted 19 March 2001

Received by V. Sgaramella

\begin{abstract}
Selective expression of enzymes that adjust the intensity of turnover of diphosphoinositolpolyphosphates may regulate vesicle trafficking and DNA repair. For example, the type 2 human diphosphoinositolpolyphosphate phosphohydrolases (hDIPP $2 \alpha$ and $2 \beta$ ) are distinguished by a solitary amino-acid residue; the type $2 \beta$ isoform contains Gln86 whereas the type $2 \alpha$ isoform does not, yet the latter has $2-5$ fold more catalytic activity than its $\beta$ counterpart (J. Biol.Chem. (2000) 12730). We discovered that both $\alpha$ and $\beta$-type mRNAs were co-expressed in clonal cell-lines. We sought a genetic explanation for this microheterogeneity. Two BACs containing distinct, but intronless, hDIPP2 $\beta$ genes were cloned. Only one of these genes could potentially give rise to our previously characterized hDIPP $2 \beta$ mRNA; the other gene has several sequence differences and, in any case, is likely a processed pseudogene. These BACS were mapped to 1q12-q21 and 1p12-p13 by FISH. No analogous intronless hDIPP $2 \alpha$ gene was detected by analysis of 21 individual genomic DNAs. However, sequence analysis of a third hDIPP2 gene (at 12q21) places the Gln86 CAG codon within an AGCAG pentamer, offering adjacent, alternate intronic 3'-boundaries. Thus, 'intron boundary skidding' by spliceosomes provides a mechanism for yielding both hDIPP $2 \alpha$ and hDIPP2 $\beta$ mRNAs. Our studies expand the repertoire of molecular mechanisms regulating diphosphoinositolpolyphosphate metabolism and function. Published by Elsevier Science B.V.
\end{abstract}

Keywords: Diphosphoinositol polyphosphate phosphohydrolases; Intron/exon; Signal transduction; Inositol phosphates

\section{Introduction}

The combinatorial manner in which phosphate groups can be arranged around the six-carbon inositol ring yields a large group of signal transduction molecules (Shears, 1998). The most highly phosphorylated members of this family are the diphosphoinositol polyphosphates (PP$\mathrm{InsP}_{4}, \mathrm{PP}-\mathrm{InsP}_{5},[\mathrm{PP}]_{2}-\mathrm{InsP}_{3}$ and $\left.[\mathrm{PP}]_{2}-\mathrm{InsP}_{4}\right)$ (Stephens et

\footnotetext{
Abbreviations: DAPI, 4',6'-Diamidino-2-phenylindole; FISH, fluorescence in situhybridization; FITC, fluorescein isothiocyanate; hDIPP $2 \alpha$ and hDIPP $2 \beta$, human diphosphoinositol polyphosphate phosphohydrolases types $2 \alpha$ and $2 \beta$; HTGS, high-throughput genomic sequence; InsP $_{6}$, inositol hexakisphosphate; nt, nucleotide; NUDT3 and NUDT4, [nucleoside diphosphate attached moiety ' $\mathrm{x}$ ']-type motif types 3 and 4, also known as the "Nudix'-type motif; PP-InsP ${ }_{4}$, diphosphoinositol tetrakisphosphate; PP$\mathrm{InsP}_{5}$, diphosphoinositol pentakisphosphate; $[\mathrm{PP}]_{2}$-InsP $\mathrm{I}_{4}$, bis-diphosphoinositol tetrakisphosphate; $[\mathrm{PP}]_{2}$-InsP $\mathrm{I}_{3}$, bis-diphosphoinositol trisphosphate; PCR, polymerase chain reaction; RH mapping, radiation hybrid mapping; RT-PCR, reverse transcriptase polymerase chain reaction; SSC, saline sodium citrate; UTR, untranslated region

* Corresponding author. Tel.: +1-919-541-0793; fax: +1-919-541-0559

E-mail address: shears@niehs.nih.gov (S.B. Shears).
}

al., 1993; Menniti et al., 1993; Shears et al., 1995; Saiardi et al., 2000). These particular molecules display a rapid rate of metabolic turnover through tightly-regulated kinase/phosphohydrolase substrate cycles that, in mammalian cells at least, are modulated by changes in levels of cytosolic $\mathrm{Ca}^{2+}$ (Glennon and Shears, 1993) and by cyclic nucleotides (Safrany and Shears, 1998). This process is interpreted as representing a multifunctional molecular switching activity (Voglmaier et al., 1996; Safrany et al., 1999; Caffrey et al., 2000) that plays important roles in diverse physiological activities, such as vesicle trafficking (Saiardi et al., 2000). Dephosphorylation of diphosphoinositol polyphosphates to InsP $_{6}$ may also regulate the efficiency of DNA repair (Hanakahi et al., 2000). Flexibility in response times of these molecular switches seem likely to depend upon factors that regulate the activities of a group of hydrolases (DIPPs) that each show distinct rates of dephosphorylation of diphosphoinositol polyphosphates (Caffrey et al., 2000). Three catalytically distinct hDIPP isoforms have so far been described: type 1 (Safrany et al., 1998), type $2 \alpha$ and type $2 \beta$ (Caffrey et al., 2000). 
The NUDT3 gene that encodes hDIPP1 has been assigned to 6p21.2-p21.33 (Safrany et al., 1998). It is more problematic to distinguish the chromosomal loci of the NUDT4 genes that encode the two hDIPP2 isoforms, because only an extremely subtle difference between the corresponding $\alpha$ and $\beta$ mRNAs is known. The $\beta$ form possesses an additional CAG codon in the coding region (Caffrey et al., 2000). Thus, the RH mapping data listed by Unigene (see Section 2.4 ), which currently offer $1 \mathrm{q} 12-21$ and $12 \mathrm{q} 21$ as potential NUDT4/hDIPP2 loci, do not distinguish whether either are type $\alpha$ or $\beta$ genes.

This is an important issue to resolve, because the additional CAG codon in the hDIPP $2 \beta$ mRNA introduces a single, in-frame amino acid residue (Gln86) into the protein. This insertion engenders the hDIPP $2 \beta$ protein with 2 - to 5 fold lower catalytic activity than its type $\alpha$ counterpart (Caffrey et al., 2000). Therefore, selective expression of the type $2 \alpha$ and $2 \beta$ enzymes can adjust the intensity of turnover and function of the diphosphoinositolpolyphosphates. An understanding of the nature of the hDIPP2 genes would be a useful step towards identifying the factors that regulate the expression of the $\alpha$ - and $\beta$-type hDIPP2 isoforms, which in turn can have an important impact upon the turnover and function of the diphosphoinositol polyphosphates. Note that the cDNA clones for both isoforms were originally amplified from a heart cDNA library that was assembled from several human donors (Caffrey et al., 2000). We report here our discovery that the two hDIPP2 isoforms are in fact co-expressed in the same clonal celltype, and we identify a possible mechanism by which this occurs.

\section{Methods}

\subsection{Northern blotting and RT-PCR}

Poly-A selected RNA was isolated from K562 and SW480 cells using an OligoTex Direct mRNA kit (Qiagen, Valencia, GA), using the manufacturer's protocol. K562 mRNA $(5 \mu \mathrm{g})$ was separated on a $1 \%$ agarose gel containing formaldehyde (NorthernMax kit; Ambion, Austin, TX), blotted and then UV-linked onto a positively charged nylon membrane. The membrane was prehybridized in digoxigenin Easy Hyb solution (Boehringer Mannheim, Indianapolis, IN) at $50^{\circ} \mathrm{C}$ for $30 \mathrm{~min}$, and probed overnight at $50^{\circ} \mathrm{C}$ with a $220 \mathrm{bp}$ PCR-generated, digoxigenin-labeled hDIPP $2 \alpha$ coding region probe ('P1', see Section 3 ). The blot was washed twice for $15 \mathrm{~min}$ in $0.1 \times \mathrm{SSC}$ at $50^{\circ} \mathrm{C}$, and bound probe was tagged with HRP-conjugated anti-digoxigenin IgG. Antibody-antigen complexes were detected by chemiluminescence using CDP-Star.

RT-PCR was programmed using $0.1 \mu \mathrm{g}$ K562 or SW480 mRNA, using the SuperScript One-Step RT-PCR system (Life Technologies, Gaithersburg, MD). In control experiments lacking reverse transcriptase, AdvanTaq Plus (Clon- tech, Palo Alto, CA) was used. The primers used were: $5^{\prime}$ GAAGTTCAAGCCCAACC-3', and 5'-CCACAGACTGATGTCAAATGGAC-3'. Reverse transcription was performed at $50^{\circ} \mathrm{C}$ for $30 \mathrm{~min}$, followed by denaturation for $2 \mathrm{~min}$ at $94^{\circ} \mathrm{C}$. PCR amplification (40 cycles) was as follows: $94^{\circ} \mathrm{C} / 15 \mathrm{~s}, 55^{\circ} \mathrm{C} / 30 \mathrm{~s}, 72^{\circ} \mathrm{C} / 2 \mathrm{~min}$. PCR products were gel-purified, and TA cloned into pCR-XL-Topo (Invitrogen, Carlsbad, CA) following the manufacturer's protocol. Individual clones were isolated and partially sequenced using dRhodamine chemistry (ABI Systems, Foster City, CA).

\subsection{BAC screening and FISH analysis}

A human BAC library was screened (Research Genetics) with ${ }^{32} \mathrm{P}$-labeled probe P2 (see Results). Two strongly hybridizing clones, 33J7 (BAC1) and 608B23 (BAC2), were identified. BAC DNA was purified using a modified DNA purification protocol and a Qiagen Plasmid Midi kit. BAC DNA was PCR cycle-sequenced using BigDye chemistry (ABI Systems). For dual-color FlSH analysis (SeeDNA Biotech, Inc., Windsor, ONT), $20 \mu \mathrm{g}$ each of BAC1 and BAC2 DNA were labeled with biotin and digoxigenin, respectively, for $2 \mathrm{~h}$ at $15^{\circ} \mathrm{C}$ (Heng and Tsui, 1993). Human lymphocyte chromosomal spreads were prepared as described (Heng and Tsui, 1993), and labeled probes were hybridized overnight in a hybridization buffer containing $50 \%$ formamide and $10 \%$ dextran sulphate. After washing, bound probes were detected using FITC (BAC1) and Rhodamine (BAC2). DAPI staining of the mitotic spreads was used to identify specific chromosomes (Heng and Tsui, 1993).

\subsection{Southern blotting}

A multiple-species genomic Southern blot (Clontech, Palo Alto, CA) was probed overnight at $37^{\circ} \mathrm{C}$ with probe P1 (see above). The blot was washed twice for $15 \mathrm{~min}$ each at $68^{\circ} \mathrm{C}$ in $0.1 \times \mathrm{SSC}$, and the bound probe detected as described above for Northern blotting.

\subsection{Sequence analysis/database searching}

All GenBank database searches were performed using the BLAST algorithim (Altschul et al., 1997), and results were analysed with the Wisconsin Package of sequence analysis software (Genetics Computer Group, Inc., Madison, WI). BAC sequences were assembled into contigs and edited using the Sequencher software package (GeneCodes Corp., Ann Arbor, MI). Mapping information for hDIPP2 (HUGO designation: NUDT4, for Nudix [nucleoside diphosphate linked moiety X] type motif 4) was obtained from Unigene (http://www.ncbi.nlm.nih.gov/UniGene/Hs.Home.html) using Hs.92381 as a cluster identifier.

\subsection{Individual genomic DNA analysis}

The 'mutagenic' upstream primer used was 5'- 
AGACTTCTGGGCATATTTGCGG-3'; the wild-type primer contains an $A$ residue in place of the underlined $C$ residue. The downstream primer used was 5'-CAAGGCATTATTATCCGG- ${ }^{\prime}$. Reactions were programmed with $100 \mathrm{pg}$ purified hDIPP $2 \alpha$ or hDIPP $2 \beta$ cDNA, or with 100 pg total genomic DNA from 21 individuals. This genomic DNA, originally obtained from the Coriell Institute for Medical Research (Camden, NJ), was provided by Dr Douglas Bell, Laboratory of Computational Biology and Risk Assessment, NIEHS. PCR was performed using a Platinum Taq DNA polymerase kit (Life Technologies, Gaithersburg, MD) and the following cycling conditions ( 35 cycles): 1 min each at 94,55 , and $72^{\circ} \mathrm{C}$. For characterization of product structure, $20 \%$ of each PCR reaction was digested with Fsp I, and the digests were separated on a $3 \%$ agarose/TBE gel.

\section{Results and discussion}

\subsection{Co-expression of $\alpha$ - and $\beta$-type hDIPP 2 mRNA in human clonal cell lines}

Northern blots of mRNA obtained from a human chronic myelogenous leukemic line (K562) revealed a major
hDIPP2 mRNA species of $4.2 \mathrm{kbp}$, and less abundant species of 1.6, 1.8, and $4.7 \mathrm{kbp}$ (Fig. 1A). Earlier studies (Caffrey et al., 2000) have shown that both $\alpha$ - and $\beta$-type mRNAs of these different sizes can be produced through utlization of an array of alternate polyadenylation motifs. RT-PCR was used to amplify K562 DIPP2 mRNA (Section 2.1). PCR products of the expected size were generated when reverse transcriptase (Fig. 1B, lane 1), but not Taq DNA polymerase (Fig. 1B, lane 3), was included in the RT-PCR reaction mix. This indicates that the amplified fragments observed are derived from mRNA, and not from possible genomic DNA contamination. In addition, no products were amplified by reverse transcription in the absence of template mRNA (Fig. 1B, lane 2). Individual PCR products were cloned and sequenced. Three PCR products contained the Gln86 CAG codon, whereas six did not. Thus, both $\alpha$ - and $\beta$-type mRNAs are present in this cell line. We extended this analysis by using mRNA from the SW480 human colonic adenocarcinoma line. Individual PCR products (Fig. 1B, lane 4) were again cloned and sequenced. In this case, three PCR products contained the Gln86 CAG codon, whereas four did not. The cellular coexpression of $\alpha$ and $\beta$ isoforms of hDIPP 2 may therefore be quite widespread.
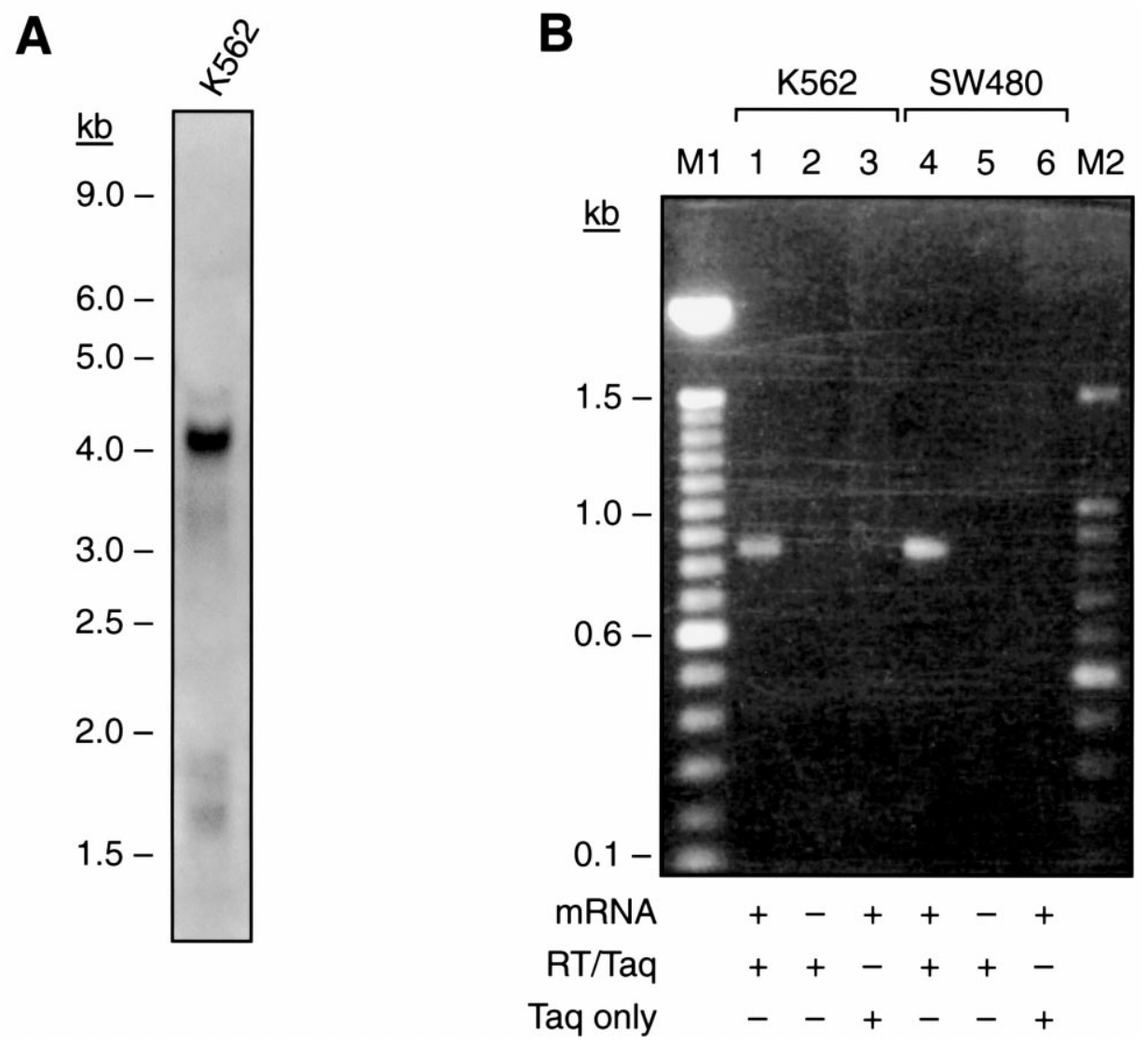

Fig. 1. Co-expression of $\alpha$ and $\beta$ mRNAs for hDIPP2 in K562 and SW480 cells. Panel A, Northern blot of $5 \mu \mathrm{g}$ polyA RNA from the cell line K562, screened with an hDIPP2 coding region probe (P1 in Fig. 3; Section 2.1). Molecular size markers are shown to the left in kilobases. Panel B, RT-PCT amplification from K562 and SW480 polyA RNA of a 858bp fragment of the hDIPP2 coding region (Section 2.1). Ten percent of each PCR reaction was electrophoresed on a $1.5 \%$ agarose gel, which was then stained with ethidium bromide. Molecular size markers are indicated in kilobases. 


\subsection{Several putative DIPP2 genes are present in the genomes of a number of species.}

We performed a Southern analysis of restriction enzymedigested genomic DNA from various species, and probed the blot with an hDIPP2 $\alpha$ coding region probe (Section 2.3). After washing the blot at high stringency $\left(0.1 \times \mathrm{SSC}\right.$ at $68^{\circ} \mathrm{C}$ for $30 \mathrm{~min}$ ), three strongly hybridizing bands and two weaker bands were detected in human DNA (Fig. 2), suggesting that more than one gene for this enzyme might exist in the genome. Moreover, even after this high stringency wash, strong hybridization to monkey, dog, cow, and rabbit genomic DNA was detected, with weaker signals in rat and mouse, indicating that DIPP2 genes are highly evolutionarily conserved.

\subsection{An hDIPP $2 \beta$-like sequence on chromosome 1 is a pseudogene}

To identify potential hDIPP2 genes we screened a human BAC library with a $1.23 \mathrm{~kb}$ cDNA probe consisting of the hDIPP $2 \alpha$ coding region, plus approximately 700 bp of the $3^{\prime}$ UTR (probe P2, see Fig. 3). This screen yielded two clones, $\mathrm{BAC} 1$ and BAC2 (Fig. 3). These were digested with restriction enzymes, Southern blotted, and the resultant blot was probed with the hDIPP $2 \alpha$ coding region probe (P1; see Fig.

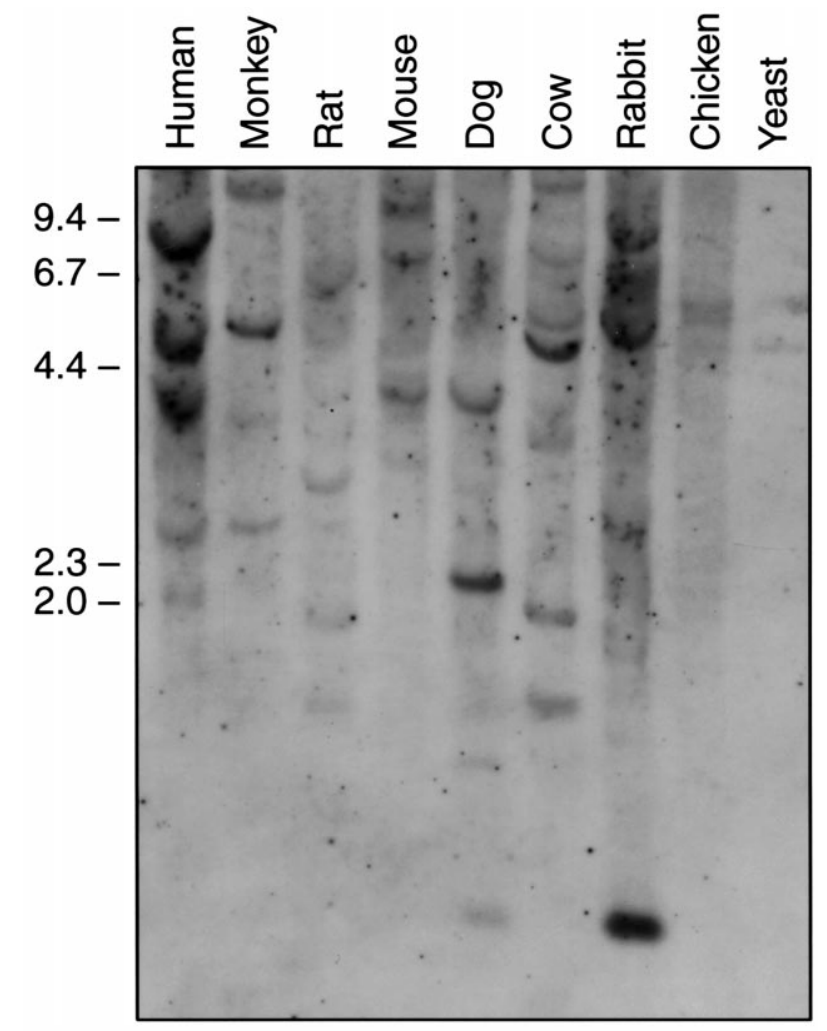

Fig. 2. Southern analysis of DIPP2 genes in different species Southern blot of $10 \mu \mathrm{g}$ EcoR I-digested genomic DNA from the indicated species, screened with a hDIPP2 coding region DNA probe (P1 in Fig. 3; Section 2.3). Note that $\mathrm{P} 1$ does not contain an Ecor I site. Molecular size markers are shown to the left in kilobases.
3). In some of the digests, two distinct patterns of hybridization were observed for each clone (data not shown), suggesting that the BACs differ from each other. A 2566 bp region of BAC1 was sequenced and found to contain an open reading frame (ORF) identical to the hDIPP2 $\beta$ coding region (Fig. 3). However, the $5^{\prime}$ UTR of this sequence in BAC1 lacks a $20 \mathrm{nt}$ GC-rich region found in the $5^{\prime}$ UTR of hDIPP2 cDNA; a separate, single nucleotide difference also distinguishes these two $5^{\prime}$ UTRs (Fig. 3, filled triangle). Furthermore, the hDIPP $2 \beta$ coding sequence in BAC1 is followed by a $3^{\prime}$ UTR that has eight single nucleotide differences (Fig. 3, filled triangles) and one $2 \mathrm{bp}$ insertion, compared to the same region of the hDIPP2 cDNA (Fig. 3). Confirmation of the nature of the BAC1 hDIPP2 $\beta$-like sequence came from its identification in a contiguous $8.5 \mathrm{kbp}$ HTGS from chromosome 1 (Accession No. AL359758; Fig. 3). The hDIPP2 $3^{\prime}$ UTR in this genomic contig terminates in a poly(A) tract just downstream of a canonical AATAAA polyadenylation signal (Fig. 3). The presence of this poly(A) tract in genomic DNA provides additional evidence that the DIPP2 $\beta$-like sequence encoded in chromosome $1 / \mathrm{BAC} 1$ is from a processed pseudogene (Mighell et al., 2000).

\subsection{Identification of a second, novel hDIPP2 $\beta$-like sequence}

Having concluded that the BAC1 hDIPP2 $\beta$-like sequence cannot account for our previously characterized hDIPP $2 \beta$ mRNA (Section 3.3), we next sequenced a 1206 bp section of BAC2, and showed that this also encoded a complete, intronless hDIPP2 $\beta$ ORF. The 601 bp $3^{\prime}$ UTR that we sequenced is equivalent to hDIPP2 mRNA, as is the $59 \mathrm{bp}$ $5^{\prime}$ UTR, which in particular includes the GC-rich region missing from BAC1 (Fig. 3). BAC2 contains a novel hDIPP2 $\beta$ gene; no corresponding HTGS clone was identified. Additional comparisons of the BAC2 and hDIPP2 cDNA were made by PCR, using 18 pairs of overlapping primers, each encompassing on average $400 \mathrm{nt}$ of the cDNA sequence, across nt 478 to 3372 (see Fig. 3). In all reactions, the single PCR product obtained using $\mathrm{BAC} 2$ as template was identical in size to that obtained using hDIPP2 cDNA as template (data not shown). Thus, the new gene in BAC2 has at least $3.5 \mathrm{kbp}$ of intronless hDIPP2 $\beta$ sequence.

\subsection{BACl and BAC2 map to opposite arms of chromosome 1}

The chromosomal locations of the BAC1 and BAC2 hDIPP2 $\beta$ genes were determined by dual-colour FISH analysis of human lymphocyte chromosome spreads. BAC1 was labeled with biotin, and detected with FITC (green/yellow dots on Fig. 4A); BAC2 was labeled with digoxigenin and detected with rhodamine (red dots on Fig. 4A). The two BACs localized to chromosome 1, with a FISH detection efficiency of 90-97\% (Fig. 4A,B). Their sequences are very similar, so each FISH probe cross-hybridized with both loci. Detailed positions were determined 
(i)

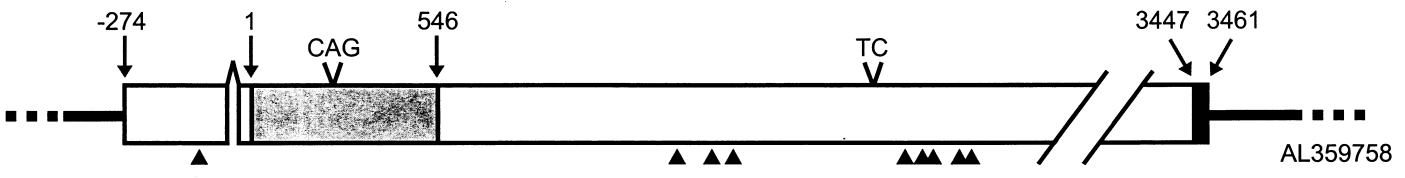

(ii)

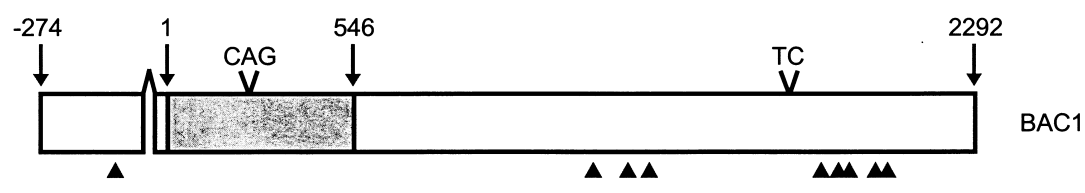

(iii)

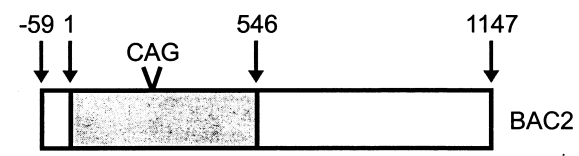

(iv)

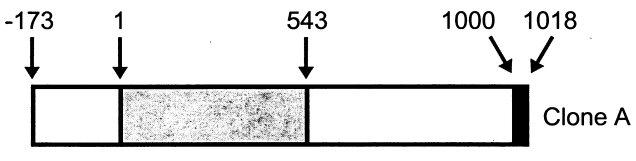

c00014 P1

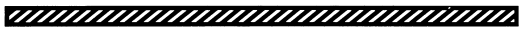

(v)

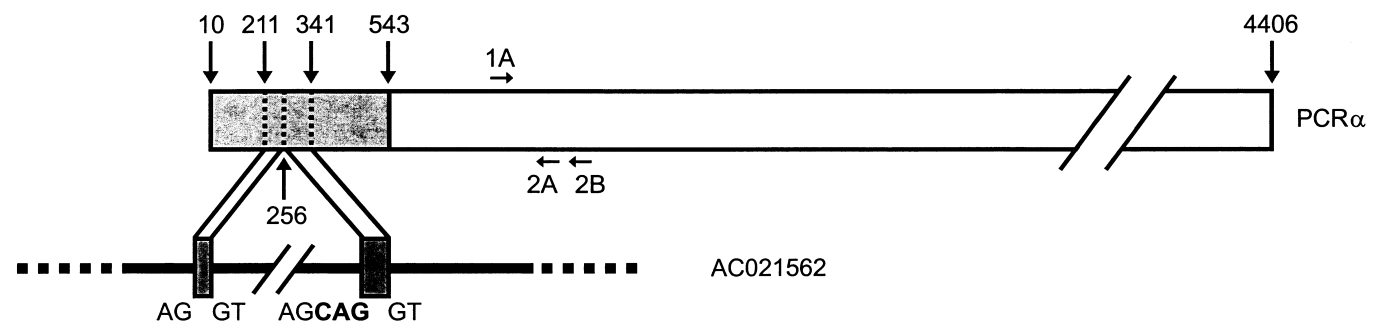

Fig. 3. hDIPP2 genes and pseudogenes The schematic shows the alignment of: (i) the hDIPP2 $\beta$ pseudogene on a contiguous section of a chromosome 1 HTGS (Accession No. AL359758); (ii) the hDIPP2 $\beta$ sequence on the BAC1 clone obtained in the current study (Accession No. AF311215); (iii) the hDIPP2 $\beta$ sequence on the BAC2 clone obtained in the current study (Accession No. AF311216); (iv) hDIPP2 $\alpha$ cDNA Clone A (Accession No. AF191649, (Caffrey et al., 2000)); (v) hDIPP2 $\alpha$ PCR product (Accession No. AF191651, (Caffrey et al., 2000)); (vi) a section of an HTGS (Accession No. AC021562), containing a likely functional hDIPP2 gene. The latter shows the putative intron/exon boundaries that provide a splice acceptor site selection process by which the CAG triplet (bold) that would encode Gln86 is either present or absent in the hDIPP2 mRNA. Nucleotides are numbered with reference to the first nucleotide of the tandem hDIPP2 initiator codons (Caffrey et al., 2000)). Coding regions are shaded grey; UTRs are white. The black bands at the ends of clones (i) and (iv) depict poly(A) tracts. Both clones (i) and (ii) contain the same eight single nucleotide differences (at positions marked by black triangles) from the previously sequenced cDNAs (clones iv and v). We do not highlight any unique features of the clone (i) sequence (such as the single nucleotide deletion in the coding region), reflecting the fact that it is a draft and likely has some sequencing errors. The horizontal arrows around clone (v), labeled 1A, 2A and 2B depict the RH mapping primers; P1 (nt 6-224) and P2 (nt 6-1234) describe the probes used for Northern/Southern analysis and BAC library screening, respectively.

based on a summary of ten photographs, revealing that the probes mapped to 1p12-p13 and 1q12-q21 (Fig. 4C). Previously, only a single DIPP2 locus on chromosome 1 (q12-q21) had been known, as a result of RH mapping data listed by Unigene (Section 2.4). Moreover, our data also provides the important, new information that neither chromosome 1 locus encodes the $\alpha$-type hDIPP2 gene.

\subsection{An hDIPP2 gene on chromosome 12 can encode both $\alpha$ and $\beta$ isoforms}

Does the human genome contain a DIPP2 $\alpha$ gene that is analogous in structure to either of the two intronless hDIPP $2 \beta$ genes? To investigate this possibility, we devised a strategy to determine the presence or absence of the Gln86 CAG codon in PCR products amplified from individual human genomic DNAs. A 'mutagenic' PCR primer containing a single base change (underlined, see below) was synthesized; this primer is designed to anneal immediately upstream of the insertion position of the Gln86 CAG codon in hDIPP2 cDNAs. The first two residues of this codon (in bold, see below), in combination with the primer mutation, create an Fsp I restriction enzyme site (TGCGCA). Selection of the downstream primer ensured that there were no other Fsp I sites in the PCR products. Thus, PCR products (264bp) derived from intronless template DNA containing the CAG encoding Gln86 will be sensitive to Fsp I digestion (generating 244bp and 20bp fragments), whereas those PCR products derived from DNA lacking the CAG codon will be resistant to Fsp I. To control for PCR amplification and restriction enzyme digestion, reactions were also performed using a wild-type upstream primer. In these reactions, irre- 
A

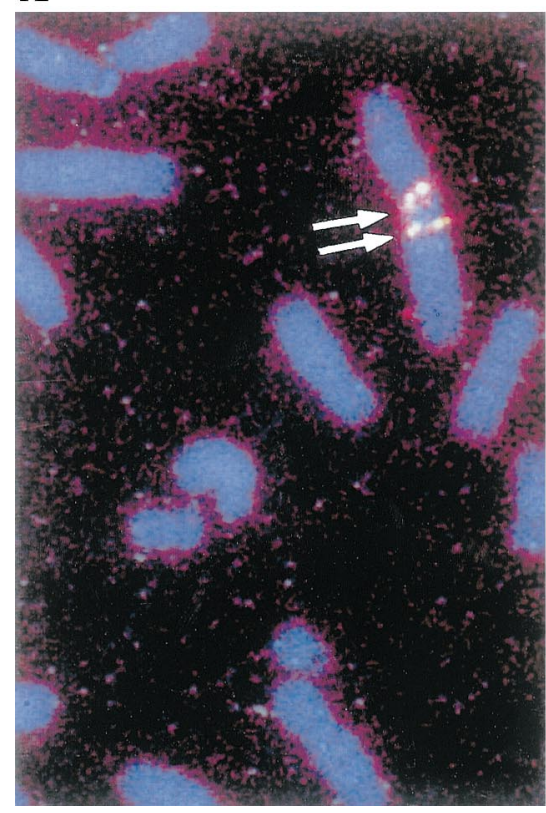

B

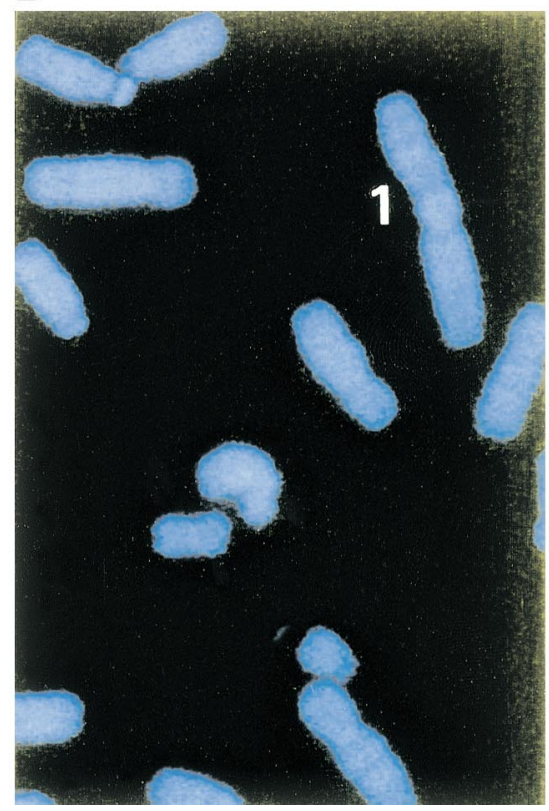

C

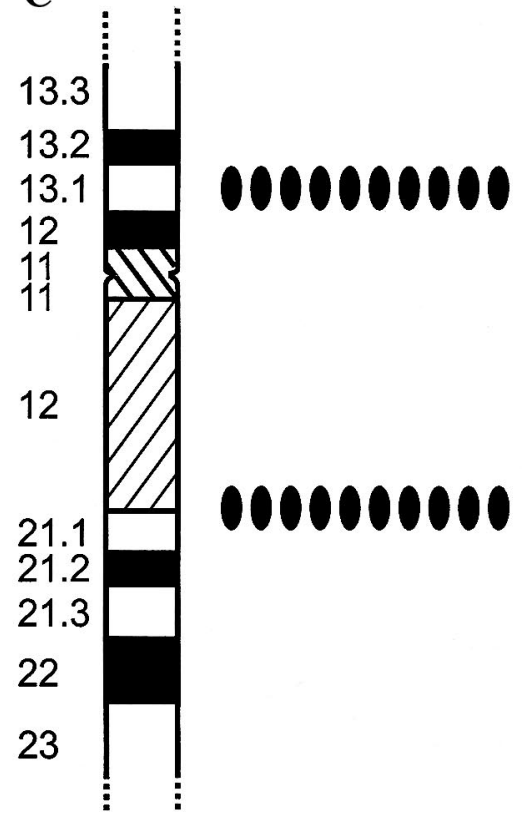

Fig. 4. hDIPP2 $\beta$ genes mapped by FISH Panel A, FISH signals (arrowed) on human chromosomes for BAC1 (green-yellow dots) and BAC2 (red dots). Panel B, The same spread as seen in Panel A, stained with DAPI to identify chromosomes. Chromosome 1 is indicated. Panel C, an ideogram of the DAPI banding pattern on the relevant section of chromosome 1. Each dot represents double FISH signals detected in the regions 1p12-p13 and 1q12-q21.

spective of the presence or absence of the Gln86 codon, a functional Fsp I site will not be generated.

PCR amplification using hDIPP $2 \alpha$ cDNA as template and the wild-type or mutagenic primers yielded a fragment of the approximate predicted size (261bp; see Fig. 5); neither fragment was sensitive to Fsp I digestion, as expected. In reactions containing hDIPP $2 \beta$ cDNA as template, the PCR product was sensitive to Fsp I digestion, but only when the mutagenic primer was used (Fig. 5). These controls confirmed that both the presence of the Gln86 codon and the use of the mutagenic primer were necessary to obtain Fsp I-digestible products. Therefore, we performed this analysis using genomic DNAs from seven individuals, and found that in all cases the PCR products were completely cleaved by Fsp I (Fig. 5). An additional 14 genomic DNAs were then screened, with identical results (data not shown). We conclude that the human genome does not contain a hDIPP $2 \alpha$ gene that lacks introns in the 261 bp region amplified by the two primers used in these PCR reactions. Presumably, the PCR products we did obtain (Fig. 5) are derived from the hDIPP $2 \beta$ gene sequences on chromosome 1 (Sections 3.3 and 3.4). There does not appear to be a hDIPP $2 \alpha$ gene that corresponds in structure to the two intronless $\beta$-type genes that we have characterized.

We therefore adopted a different strategy to search for a hDIPP $2 \alpha$ gene. We performed three independent $\mathrm{RH}$ mapping experiments on the Stanford G3 panel, using two different pairs of hDIPP2 $3^{\prime}$ UTR primers $(1 \mathrm{~A} / 2 \mathrm{~A}$, once; 1A/2B, twice; see Fig. 3). In each experiment, linkage to the ordered marker SHGC-33476 on 12q21 was predicted, with LOD scores of 7.1, 7.7 and 8.0. Furthermore, we iden- tified two overlapping HTGS clones that contain this hDIPP2 gene, AC021562 and AC025260. (Although HTGS AC021562, version 3, is tentatively assigned by GenBank to chromosome 11, it overlaps with HTGS

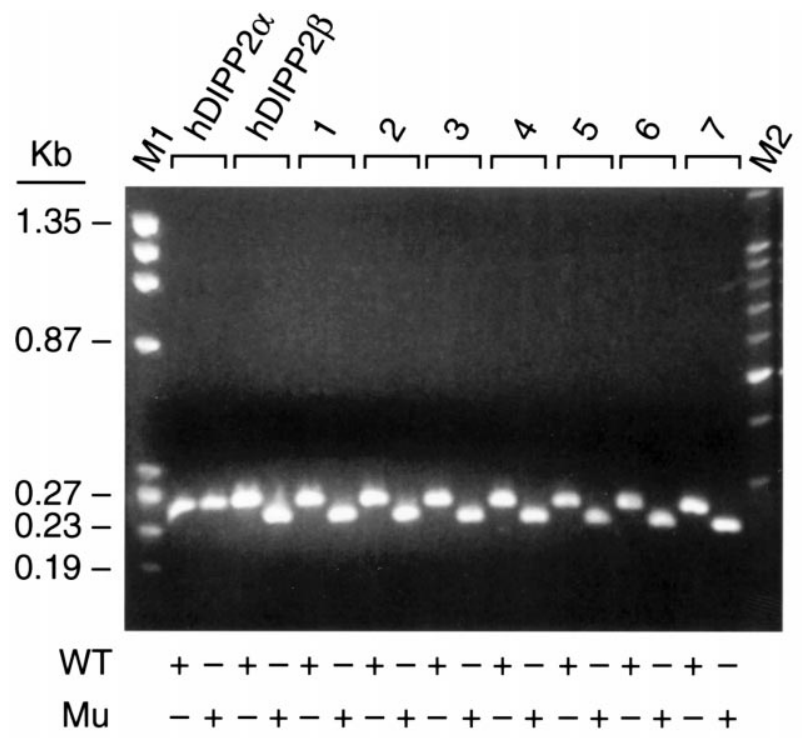

Fig. 5. Analysis of hDIPP2 genes in individual genomic DNAs The figure shows agarose (3\%) gel analysis of Fsp I-digested PCR products. Two PCR reactions were programmed with either hDIPP $2 \alpha$ or hDIPP2 $\beta$ cDNA templates (as indicated), or with total genomic DNA from a panel of individuals (lanes 1-7) (Section 2.5). For each template, the reactions were primed with either the wild-type (WT) or mutagenic $(\mathrm{Mu})$ upstream primer, as indicated beneath the figure. M1 and M2 are molecular size markers. Sizes are indicated to the left in kilobases $(\mathrm{kb})$. 
Table 1

Summary of hDIPP2 loci identified in the current study ${ }^{\mathrm{a}}$

\begin{tabular}{ll}
\hline Locus & hDIPP2 subtype \\
\hline $1 \mathrm{q} 12-\mathrm{q} 21$ & type $\beta$ \\
$1 \mathrm{p} 12-\mathrm{p} 13$ & type $\beta$ \\
$12 \mathrm{q} 21$ & type $\alpha$ and $\beta$ can arise by intron \\
& boundary skidding \\
\hline
\end{tabular}

a Refer to the text for evidence that at least one and possibly both of the chromosome 1 loci are pseudogenes.

AC025260, version 18, which is itself tentatively assigned by GenBank to chromosome 12. The latter assignment is also in agreement with both our RH mapping data, and data listed by Unigene, see Section 2.4). Almost 95\% of our previously described hDIPP2 cDNA (PCR $\alpha /$ clone (v), see Fig. 3) is present in clones AC021562 and AC025260. We partioned the DNA sequences into four exons (data not shown), each of which is bracketed by an AG/GT consensus boundary (Anderson and Moore, 1997). The AG dinucleotide at the $3^{\prime}$ end of each putative intron occurs in an appropriate context, i.e., it is preceded by $10-15 n t$ of a predominantly polypyrimidine tract (Anderson and Moore, 1997). It is particularly significant that the $3^{\prime}$ boundary of one putative intron comprises an AGCAG pentamer that provides tandem, alternate $3^{\prime}$ splice acceptor sites (Fig. 3). Depending upon which acceptor site is chosen, a CAG codon (bold, Fig. 3), encoding Gln86, will be present or absent in the final mRNA. Previous studies have shown that, when only $3 \mathrm{nt}$ separates two splice acceptor sites, either may be chosen with approximately equal efficiency, (Nakamura et al., 1997; Lin et al., 2000). This flexibility in choice of acceptor site can be described as 'intron boundary skidding'. Thus, we have discovered that the gene on chromosome 12 can potentially encode both hDIPP $2 \alpha$ and $\beta$ isoforms, by alternate splicing of a single transcript. The intron/exon structure of this gene (Fig. 3) is such that it would not have been detected by the mutagenic primer strategy described above.

\subsection{Concluding statement}

If we are to identify the factors that regulate the turnover and hence the function of diphosphoinositol polyphosphates, we need to understand the structure of the NUDT genes that encode the various hDIPP isoforms. Previous work has not attempted to distinguish genes that might give rise to the catalytically-distinct type $\alpha$ and $\beta$ isoforms of hDIPP2. We have now characterized two NUDT4/ hDIPP $2 \beta$ loci which are adjacent to, and on opposite sides of, the chromosome 1 centromere (Table 1). Based on the intronless nature of these genes, and additional sequence analysis, we conclude that at least one of them is a processed pseudogene. A third hDIPP2 locus was mapped to chromosome 12 , from which both type $\alpha$ and $\beta$ mRNAs could be transcribed (Table 1).
It is particularly significant that both $\alpha$ and $\beta$ isoforms can be co-expressed in single cells. This DIPP2 microheterogeneity is apparently quite widespread, since ESTs for DIPP2 $\alpha$ and DIPP2 $\beta$ have been also obtained from mice and rats (Caffrey et al., 2000). Our studies into the genetic rationale for such microheterogenity adds to the repertoire of molecular mechanisms that the cell has at its disposal for regulating overall DIPP expression. Other processes include the generation of multiple mRNA transcripts of differing lengths, even in clonal cell lines (Fig. 1A; Caffrey et al., 2000), that may differ in stability and/or translatability. In addition to hDIPP $2 \alpha$ and hDIPP $2 \beta$, further catalytic diversity is provided by type 1 (Safrany et al., 1998) and type 3 (J.J. Caffrey and S.B. Shears, unpublished data) isoforms of hDIPP. We (Caffrey et al., 2000) have previously proposed that these different opportunities to carefully tailor cellular expression of DIPP activity provide tight regulation of the response times of the molecular switching activities that are believed to underlie the physiological significance of diphosphoinositol polyphosphates.

\section{Acknowledgements}

We thank Dr John York (Howard Hughes Medical Institute, Duke University, NC) for generous help with RH mapping.

\section{References}

Altschul, S.F., Madden, T.L., Schaffer, A.A., Zhang, J., Zhang, Z., Miller, W., Lipman, D.J., 1997. Gapped BLAST and PSI-BLAST: a new generation of protein database search programs. Nucleic Acids Res. 25, 3389-3402.

Anderson, K., Moore, M.J., 1997. Bimolecular exon ligation by the human spliceosome. Science 276, 1712-1716.

Caffrey, J.J., Safrany, S.T., Yang, X., Shears, S.B., 2000. Discovery of molecular and catalytic diversity among human diphosphoinositol polyphosphate phosphohydrolases: an expanding NUDT family. J. Biol. Chem. 275, 12730-12736.

Glennon, M.C., Shears, S.B., 1993. Turnover of inositol pentakisphosphates, inositol hexakisphosphate and diphosphoinositol polyphosphates in primary cultured hepatocytes. Biochem. J. 293, 583-590.

Hanakahi, L.A., Bartlet-Jones, M., Chappell, C., Pappin, D., West, S.C., 2000. Binding of inositol phosphate to DNA-PK and stimulation of double-strand break repair. Cell 102, 721-729.

Heng, H.H.Q., Tsui, L.-C., 1993. Modes of DAPI banding and simultaneous in situ hybridization. Chromosoma 102, 325-332.

Lin, M.-J., Lee, T.-L., Hsu, D.-W., Shen, C.-K.J., 2000. One codon alternative splicing of the CpG MTase Dnmt1 transcript in mouse somatic cells. FEBS Lett. 469, 101-104.

Menniti, F.S., Miller, R.N., Putney Jr, J.W., Shears, S.B., 1993. Turnover of inositol polyphosphate pyrophosphates in pancreatoma cells. J. Biol. Chem. 268, 3850-3856.

Mighell, A.J., Smith, N.R., Robinson, P.A., Markham, A.F., 2000. Vertebrate pseudogenes. FEBS Lett. 468, 109-114.

Nakamura, T.M., Morin, G.B., Chapman, K.B., Weinrich, S.L., Andrews, W.H., Lingner, J., Harley, C.B., Cech, T.R., 1997. Telomerase catalytic subunit homologs from fission yeast and human. Science 277, 955-959. 
Safrany, S.T., Shears, S.B., 1998. Turnover of bis-diphosphoinositol tetrakisphosphate in a smooth muscle cell line is regulated by $\beta_{2}$-adrenergic receptors through a cAMP-mediated, A-kinase-independent mechanism. EMBO J. 17, 1710-1716.

Safrany, S.T., Caffrey, J.J., Yang, X., Bembenek, M.E., Moyer, M.B., Burkhart, W.A., Shears, S.B., 1998. A novel context for the "MutT" module, a guardian of cell integrity, in a diphosphoinositol polyphosphate phosphohydrolase. EMBO J. 17, 6599-6607.

Safrany, S.T., Caffrey, J.J., Yang, X., Shears, S.B., 1999. Diphosphoinositol polyphosphates: the final frontier for inositide research? Biol. Chem. 380, 945-951.

Saiardi, A., Caffrey, J.J., Snyder, S.H., Shears, S.B., 2000. The Inositol Hexakisphosphate Kinase Family: Catalytic Flexibility, and Function in Yeast Vacuole Biogenesis. J. Biol. Chem. 2000, 275-24686.
Shears, S.B., 1998. The versatility of inositol phosphates as cellular signals. Biochim. Biophys. Acta 1436, 49-67.

Shears, S.B., Ali, N., Craxton, A., Bembenek, M.E., 1995. Synthesis and metabolism of bis-diphosphoinositol tetrakisphosphate in vitro and in vivo. J. Biol. Chem. 270, 10489-10497.

Stephens, L.R., Radenberg, T., Thiel, U., Vogel, G., Khoo, K.-H., Dell, A., Jackson, T.R., Hawkins, P.T., Mayr, G.W., 1993. The detection, purification, structural characterization and metabolism of diphosphoinositol pentakisphosphate(s) and bisdiphosphoinositol tetrakisphosphate(s). J. Biol. Chem. 268, 4009-4015.

Voglmaier, S.M., Bembenek, M.E., Kaplin, A.I., Dormán, G., Olszewski, J.D., Prestwich, G.D., Snyder, S.H., 1996. Purified inositol hexakisphosphate kinase is an ATP synthase: diphosphoinositol pentakisphosphate as a high-energy phosphate donor. Proc. Natl. Acad. Sci. USA 93, 4305-4310. 